\title{
In need of counseling?
}

\section{Moves to clamp down on companies offering direct-to-consumer genetic tests are out of step with personal genomics and its potential to empower individuals' role in their own healthcare.}

For tend or many, an intrinsic curiosity about self drives the natural human tendency towards science. If there is information to be had, especially information about that subject most important to our egosour individual selves—-we want it. Curiosity is very 'me'-driven. What more natural subject material is there for people than the very fabric of their being - their genome, their health?

So when it becomes possible to have part of your genome sequenced for less than a few thousand dollars, it is hardly surprising that people who believe that the genome provides signposts to future health flock

을 to companies like $23 \mathrm{andMe}$, deCODEme and Navigenics or any of the other haplotyping and genome resequencing companies to have that work done.

The problem is that most of the medical profession seems completely unprepared for the onslaught. And so does the existing system of legal and regulatory oversight.

In April, officials at the New York State Department of Health issued notices to twelve gene-testing firms to cease and desist from providing genome information to the public unless tests had been ordered by doctors. In mid-June, officials in California followed suit. Across the ocean, the Council of Europe has also moved to censure commercial gene testing (see p. 736).

In essence, the clampdown by lawmakers is based on technicalities: the regulators are not saying that the genetic information provided directly to the consumer is necessarily inaccurate, misleading or useless-all of which might be true to varying degrees-but simply that the laboratories providing it are either not registered under the Clinical Laboratory Improvement Amendments regulations and/or that the tests have not been ordered by a medical professional. This is somewhat like prosecuting $\mathrm{Al}$ Capone for tax evasion, a misstep on the part of the miscreant but hardly the crux of the issue.

The legitimate concern is that providing people with information on their own DNA, unless interpreted through the medium of a health professional or genetic counselor, will lead to undue worries or rash healthcare decisions on behalf of the informed person. On balance, the argument goes, it is preferable for people to remain ignorant about their personal genome than to let the 'genome' out of the bottle.

Certainly, the medical establishment seems to be united in its defense of the regulators' actions and in their distaste for this new fad of personal genome exploration. In an editorial earlier this year, The New England Journal of Medicine (358, 105-107, 2008) objects to the circumvention of the physician by direct-to-consumer services. In essence, the editors urge their medical readership to discourage patients who come into their offices brandishing their genome data or asking for help in interpreting them. Various US medical associations also hold the same line and highlight a need for guidelines on the use of tests, standards for laboratory practice and accreditation of laboratories. Others point to ethical risks, such as genetic discrimination, implications for family members, privacy and disposal of the sample. They call for genetic counselors to handle the delicate task of conveying the implications of the tests to the test subject.

On the other hand, libertarians argue that if individuals wish to explore their own genome, then it is their information to explore and no government or medical body has a right to deny them access.

But the real concern is that many of the present objections overlook the vast scale of the challenge facing healthcare in the coming decades. It is virtually impossible to conceive of a sustainable form of healthcare that operates as the current systems in industrialized nations do. At present, healthcare is based on the late diagnosis of disease and the division of diseases into a few categories based on some overarching gross similarities. And it firmly places physicians as the central gatekeepers of information.

The healthcare of the future, on the other hand, if the technical potential to provide personalized medicine is ever to be realized, will probably require a greatly expanded emphasis on diagnosis and monitoring, early and subtle intervention, monitoring of the impact of intervention, and gradual adaptation of treatment with the evolving physiology, metabolism and lifestyle of the individual.

Faced with this huge expansion of data on 'my' health, it will simply not be affordable to maintain our dependence on medical gatekeepers, whether they are physicians or genetic counselors, without individuals taking a much greater responsibility for their own wellbeing. Whether the medical establishment likes it or not, it will be too cumbersome and too expensive to conduct personalized medicine if all diagnostic-to-therapeutic decisions depend on doctors.

The transition from general healthcare advice dispensed on the basis of population data to very specific diagnosis and treatment that draws on individual data is what will transform healthcare. This means that there will need to be some stratification of what type of genetic testing requires medical oversight and counseling and what does not.

For personal genomics not to be stillborn, the medical community and regulators thus need to reevaluate their role as gatekeepers. Clearly, they need to be involved in the medical actions that might follow as a consequence of genetic or other diagnostic testing. And for any gene test, regulators must ensure that companies make claims to consumers that are both truthful and accurate. But simply shutting down the whole direct-to-consumer gene testing enterprise because it departs from the traditional genetic testing paradigm of doctorordered test will both retard progress and stifle investment in more advanced whole-genome sequencing technologies-technologies that have the potential to ultimately deliver the promise of genomedirected medicine. 